Samuel, M. S., Rath, N., Masre, S. F., Boyle, S. T., Greenhalgh, D. A., Kochetkova, M., Bryson, S., Stevenson, D. and Olson, M. F. (2016) Tissue-selective expression of a conditionally-active ROCK2-estrogen receptor fusion protein. Genesis, 54(12), pp. 636-646.

(doi:10.1002/dvg.22988)

There may be differences between this version and the published version. You are advised to consult the publisher's version if you wish to cite from it.

This is the peer-reviewed version of the following article: Samuel, M. S., Rath, N., Masre, S. F., Boyle, S. T., Greenhalgh, D. A., Kochetkova, M., Bryson, S., Stevenson, D. and Olson, M. F. (2016) Tissue-selective expression of a conditionally-active ROCK2-estrogen receptor fusion protein. Genesis, 54(12), pp. 636-646, which has been published in final form at 10.1002/dvg.22988. This article may be used for noncommercial purposes in accordance with Wiley Terms and Conditions for Self-Archiving.

\title{
http://eprints.gla.ac.uk/130871/
}

Deposited on: 02 November 2016 


\section{Tissue-selective expression of a conditionally-active ROCK2-estrogen receptor}

\section{fusion protein}

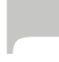

Michael S Samuel ${ }^{1,2}$, Nicola Rath ${ }^{3}$, Siti F Masre ${ }^{4,5}$, Sarah T. Boyle ${ }^{1}$, David A

Greenhalgh $^{5}$, Marina Kochetkova ${ }^{1}$, Sheila Bryson ${ }^{3}$, David Stevenson ${ }^{3}$, Michael F

$\sqrt{1}$

$$
\text { Olson }^{3,6}
$$

${ }^{1}$ Centre for Cancer Biology, SA Pathology and the University of South Australia,

Frome Road, Adelaide 5000, Australia

${ }^{2}$ School of Medicine, Faculty of Health Sciences, University of Adelaide, Adelaide SA

5000 , Australia

${ }^{3}$ Cancer Research UK Beatson Institute, Garscube Estate, Switchback Road,

Glasgow G61 1BD, UK

${ }^{4}$ Biomedical Science Programme, School of Diagnostic and Applied Health Sciences, Faculty of Allied Health Sciences, University of Kebangsaan, 50300 Kuala Lumpur, Malaysia

${ }^{5}$ Section of Dermatology and Molecular Carcinogenesis, College of Medical, Veterinary and Life Sciences, University of Glasgow, Glasgow G12 8QQ, UK ${ }^{6}$ Institute of Cancer Sciences, University of Glasgow, Glasgow G12 8QQ, UK

Correspondence to Michael F Olson

Cancer Research UK Beatson Institute, Garscube Estate, Switchback Road, Glasgow G61 1BD, UK

Tel: +44 (0)1413303654 Fax: +44(0)1419426521

Email: m.olson@beatson.gla.ac.uk

This article has been accepted for publication and undergone full peer review but has not been through the copyediting, typesetting, pagination and proofreading process which may lead to differences between this version and the Version of Record. Please cite this article as an 'Accepted Article', doi: 10.1002/dvg.22988

(C) 2015 Wiley Periodicals, Inc.

Received: Jul 28, 2016; Revised: Oct 12, 2016; Accepted: Oct 20, 2016 


\section{Abstract}

The serine/threonine kinases ROCK1 and ROCK2 are central mediators of actomyosin contractile force generation that act downstream of the RhoA small GTPbinding protein. As a result, they have key roles in regulating cell morphology and proliferation, and have been implicated in numerous pathological conditions and diseases including hypertension and cancer. Here we describe the generation of a gene-targeted mouse line that enables CRE-inducible expression of a conditionallyactive fusion between the ROCK2 kinase domain and the hormone-binding domain of a mutated estrogen receptor (ROCK2:ER). This two-stage system of regulation allows for tissue-selective expression of the ROCK2:ER fusion protein, which then requires administration of estrogen analogues such as tamoxifen or 4hydroxytamoxifen to elicit kinase activity. This conditional gain-of-function system was validated in multiple tissues by crossing with mice expressing CRE recombinase under the transcriptional control of cytokeratin14 (K14), murine mammary tumor virus (MMTV) or cytochrome P450 Cyp1A1 (Ah) promoters, driving appropriate expression in the epidermis, mammary or intestinal epithelia respectively. Given the interest in ROCK signaling in normal physiology and disease, this mouse line will facilitate research into the consequences of ROCK activation that could be used to complement conditional knockout models.

\section{Introduction}

The actomyosin cytoskeleton provides the physical structure that determines cell morphology; dynamic changes in cytoskeleton architecture are responsible for alterations in cell shape, adhesion, migration and division. Members of the Rho family of small molecular weight GTP-binding proteins (Rho GTPases) are key 
regulators of cytoskeleton organization (Olson, 2016; Sadok and Marshall, 2014). Filamentous actin (F-actin) fibers associate with myosin motor proteins that hydrolyze ATP to power cytoskeletal contractile force generation (Geeves, 2016). Amongst the most important mediators of Rho GTPase signaling that influence contractile force are the serine/threonine kinases ROCK1 and ROCK2, which phosphorylate myosin regulatory light chains leading to increased myosin ATPase activity and consequent association with F-actin (Geeves, 2016; Julian and Olson, 2014; Rath and Olson, 2012).

There has been considerable interest in the biological functions of the ROCK kinases because of their apparent promise as chemotherapeutic targets in a variety of diseases and pathological conditions. The first described ROCK inhibitor Y27632 was discovered based on its ability to reverse agonist-induced contraction of smooth muscle, including aortic, mesenteric and coronary artery tissue samples, and to lower the blood pressure of hypertensive rats (Uehata et al., 1997). Fasudil is a potent ROCK inhibitor, and has been routinely used in Japan to reduce the risk of cerebral vasospasm associated with subarachnoid hemorrhage (Satoh et al., 2014). It has also been suggested that ROCK inhibitors could be used for cancer therapy to reduce primary tumor growth and to combat metastatic dissemination (Olson, 2008; Rath and Olson, 2012). Recently, a multi-AGC kinase inhibitor AT13148 that has been in Phase I clinical testing was shown to be a potent ROCK inhibitor with antitumor activity that also blocked melanoma cell migration and metastasis in vivo (Sadok et al., 2015; Yap et al., 2012).

Complete knockouts of Rock1 or Rock2 revealed important roles in the movement of epithelial sheets necessary for eyelid and ventral body wall closure and for the closure of umbilical rings (Shimizu et al., 2005; Thumkeo et al., 2005). Mice 
that allow for the conditional knockout of Rock1 or Rock2 have been generated and used to examine how ROCK1 contributes to specific biological processing including reactive fibrosis in response to heart pressure overload (Zhang et al., 2006) and insulin receptor signaling (Lee et al., 2009). By combining conditional deletion of Rock1 and Rock2, it was determined that together they make essential contributions to cell cycle progression, and have redundant and obligatory roles in non-small cell lung cancer and melanoma (Kümper et al., 2016). These loss-of-function approaches can be very informative about the requirement for ROCK signaling in various contexts, and help to predict and validate the effect of inhibitor administration.

As a complement to loss-of-function systems or the use of pharmacological inhibitors to study ROCK signaling, we constructed conditionally-active forms of ROCK1 and ROCK2 by fusing the kinase domains with the hormone-binding domain of the estrogen receptor (ER) (Croft et al., 2005; Croft et al., 2004; Croft and Olson, 2006a). By treating cells with estrogen analogues including tamoxifen or 4hydroxytamoxifen (4HT), it is possible to switch on ROCK2:ER fusion protein activity in a time and dose-dependent manner (Croft and Olson, 2006a). This conditional activation system has been used to study ROCK signaling and consequent actomyosin contractility in cell lines in vitro and in vivo (Croft et al., 2005; Croft et al., 2004; Croft and Olson, 2006a; Croft and Olson, 2006b; Kumar et al., 2012; SanzMoreno et al., 2011). The isolated kinase domains used in the ER-fusion proteins are similar to the fragments produced by caspase (Coleman et al., 2001) or granzyme B mediated proteolysis (Sebbagh et al., 2005), as well as examples of truncated protein products that result from somatic mutations in human tumors (Lochhead et al., 2010). To examine the role of ROCK activation in vivo, we generated a 
genetically engineered mouse line in which expression of a ROCK2:ER fusion protein was driven by the cytokeratin 14 (K14) promoter (Samuel et al., 2009). Conditional activation of ROCK2:ER was sufficient to induce epidermal hyperplasia and the conversion of chemically-induced cutaneous papillomas to invasive carcinomas (Samuel et al., 2011) and also to accelerate the healing of full thickness skin wounds (Kular et al., 2015).

To extend the utility of the conditionally-active ROCK2:ER system for gain-offunction studies including in development, we generated a genetically modified mouse line by targeting the Hypoxanthine phosphoribosyltransferase (Hprt) locus on the $\mathrm{X}$ chromosome with a construct in which ROCK2:ER transgene transcription from an artificial chicken actin gene (CAG) promoter (Niwa et al., 1991) is dependent on CRE recombinase-mediated removal of a quadruple SV40 polyadenylation sequence transcriptional "stop" cassette flanked by loxP sites (Jackson et al., 2001). By crossing with mice expressing CRE recombinase from a $K 14$ promoter, we found that it was possible in mouse epidermal keratinocytes to induce expression of the ROCK2:ER fusion protein that could then be conditionally activated with $4 \mathrm{HT}$. This genetically modified mouse line will allow future studies aimed at characterizing the effects of ROCK gain-of-function in a tissue-specific manner to complement existing conditional Rock1 and Rock2 deletion mice.

\section{Results and Discussion}

To establish a conditionally active ROCK2 fusion protein (Figure 1A), a DNA sequence encoding the kinase domain (amino acids 5-553) of human ROCK2 was joined to that encoding enhanced green fluorescent protein (EGFP) at the 5 ' end and an engineered version of the hormone binding domain of the estrogen receptor $(E R)$ 
at the 3' end, modified to include a G525R replacement that greatly reduces binding of $17 \beta$ estradiol, to prevent activation by circulating estrogenic hormone, while retaining binding of $4 \mathrm{HT}$ (Danielian et al., 1993) to allow activation by exogenous ligands. When treated with $4 \mathrm{HT}$, conditional ROCK2:ER activation leads to the phosphorylation of substrates including the regulatory myosin light chains (MLC), the myosin binding subunit (MYPT) of the MLC phosphatase complex and LIM kinases 1 and 2 (LIMK1/2), which collectively results in actomyosin contractile force generation (Croft and Olson, 2006a). As a control for expression of the ROCK2:ER fusion protein that might have additional non-catalytic functions, we also made a kinasedead version (KD2:ER) that has a single amino acid mutation (K125G) that blocks ATP-binding and kinase catalytic activity (Croft and Olson, 2006a).

HM1 embryonic stem cells lack the promoter and first two exons of the Hprt locus on the X-chromosome (Selfridge et al., 1992). By targeting this locus with constructs containing flanking homology arms that include the human HPRT promoter and first exon as well as the second and third exon of mouse Hprt, it is possible to reconstitute a functional Hprt gene that enables positive selection of successful recombinants in hypoxanthine-aminopterin-thymidine (HAT) medium (Bronson et al., 1996). In this way, we were able to specifically target the ROCK2:ER transgene to the Hprt locus (Figure 1B).

To allow for conditional expression of ROCK2:ER, the fusion protein coding sequence was cloned downstream of a synthetic promoter (CAG) consisting of a cytomegalovirus early enhancer element, a chicken $\beta$-actin gene promoter with the first exon and intron, and a splice acceptor (SA) from the rabbit $\beta$-globin gene (Niwa et al., 1991), followed by a loxP-Stop-loxP (LSL) transcriptional stop cassette (Figure 1B). The inclusion of quadruple SV40 derived polyadenylation signals (Stop) within 
this cassette leads to the premature termination of transcription, until CRE-mediated recombination of the loxP sites removes the Stop cassette to allow transcription to proceed (Figure 1C). By crossing with mice expressing CRE recombinase from tissue selective promoters, it would be possible to restrict ROCK2:ER expression to targets of choice, thereby enabling for determination of the consequences of ROCK activation in a site-specific manner.

To validate the inducibility of ROCK2:ER expression in vivo, LSL-ROCK2:ER mice were intercrossed with mice expressing a fusion protein comprising CRE recombinase and the hormone binding domain of the progesterone receptor $(P R)$ under the control of the $K 14$ promoter (Figure $2 \mathrm{~A}$ ), which allows for conditional CRE activation by ligands including RU486 (Berton et al., 2000) and expression of the ROCK2:ER fusion protein. Subsequent treatment with 4-hydroxytamoxifen would then activate the expressed ROCK2:ER fusion protein (Figure 2A). Polymerase chain reaction (PCR) analysis of DNA isolated from ear skin that had been topically treated with RU486 ( $2 \mu \mathrm{g}$ in $20 \mu$ l ethanol per ear for 1 week) demonstrates the CRE and ligand-dependent recombination of $L S L-R O C K 2: E R$ (Figure 2B). In all cases, a LSL-ROCK2:ER 575 base pair (bp) PCR product was visible (Figure 2B, top and bottom panels), while a 600 bp CRE:PR transgene product was evident in the 4 positive samples (Figure 2B, middle panel). Only in the presence of both CRE:PR and RU486 treatment was there loxP-Stop-loxP recombination that led to the production of a 500 bp ROCK2:ER band (Figure 2B, lower panel). Furthermore, immunohistochemical (IHC) staining of untreated or RU486 treated ear skin with antibodies against EGFP (Figure 2C) or ROCK2 (Figure 2D) revealed ROCK2:ER expression restricted to the epidermal layer following CRE:PR stimulation with RU486. In mouse skin treated with RU486 to permit ROCK2:ER expression, the 
additional topical application of $4 \mathrm{HT}(330 \mu \mathrm{g}$ in $20 \mu \mathrm{l}$ solution in ethanol) resulted in inCREased phosphorylation of the ROCK substrate MYPT1 on T696 (pMYPT1) (Figure 2E). Further confirmation of the CRE-dependent expression of ROCK2:ER was obtained by crossing $L S L-R O C K 2: E R$ mice with a mouse line that expressed constitutively active CRE from a K14 promoter (Dassule et al., 2000) (Figure 2F). When keratinocytes were isolated from mouse tails and cultured in vitro as we previously described (Samuel et al., 2011), western blotting of cell lysates revealed that ROCK2:ER expression in LSL-ROCK2:ER mice was only observed in the presence of K14-CRE, similar to the ROCK2:ER expression in the K14-ROCK2:ER positive control keratinocytes (Samuel et al., 2009). Similar to previous observations (Croft et al., 2004), treatment with $1 \mu \mathrm{M} 4 \mathrm{HT}$ increased the levels of ROCK2:ER protein in K14-CRE; LSL-ROCK2:ER and K14-ROCK2:ER keratinocytes (Figure 2F), likely through protein stabilization. These results confirm CRE-induced ROCK2:ER expression in LSL-ROCK2:ER mice in cytokeratin 14 positive epidermal keratinocytes.

To further validate the tissue-specific inducibility and functionality of ROCK2:ER in target tissues, the LSL-ROCK2:ER and LSL-KD2:ER mice were separately crossed onto the Tg(MMTV-CRE)4Mam/J (MMTV-CRE) (Wagner et al., 1997) and Tg(Cyp1a1-CRE)1Dwi (Ah-CRE) (Ireland et al., 2004) strains, which express CRE recombinase within the mammary and intestinal epithelia respectively (Figure 3A, 4A). These crosses yielded the further strains MMTV-CRE; LSLROCK2:ER, MMTV-CRE; LSL-KD2:ER, Ah-CRE; LSL-ROCK2:ER and Ah-CRE; LSL-KD2:ER. To determine whether ROCK2:ER could be expressed and conditionally activated within the mammary epithelium, twice weekly intra-peritoneal injections of tamoxifen (60 mg/kg body weight in corn oil) were administered to 
cohorts of 8 week old LSL-ROCK2:ER, LSL-KD2:ER, MMTV-CRE; LSL-ROCK2:ER and MMTV-CRE; LSL-KD2:ER mice. Mice were sacrificed 24 hours after the final injection and sections derived from formalin-fixed paraffin-embedded mammary tissue were subjected to immunofluorescence analysis, revealing expression of the ROCK2:ER or KD:ER fusion proteins in MMTV-CRE; LSL-KD2:ER and MMTV-CRE; LSL-ROCK2:ER tissues (Figure 3B), accompanied by enhanced phosphorylation of the ROCK target MYPT1 at T696 in MMTV-CRE; LSL-ROCK2:ER mouse mammary epithelium but not in the MMTV-CRE; LSL-KD2:ER tissue (Figure 3C).

To determine whether ROCK2:ER could be expressed and conditionally activated within the intestinal epithelium, four intra-peritoneal injections of $\beta$ Naphthoflavone (80 mg/kg body weight per injection) were administered to cohorts of 8 week old $A h-C R E ;$ LSL-KD2:ER and Ah-CRE; LSL-ROCK2:ER mice over 48 hours to induce intestine-specific CRE recombinase expression. Four days following the final injection of $\beta$-Naphthoflavone, mice were injected intraperitoneally with tamoxifen (60 mg/kg body weight in corn oil), with a further injection of tamoxifen a week later. Mice were sacrificed 24 hours after the final injection and sections derived from formalin-fixed paraffin-embedded intestinal tissue were subjected to immunofluorescence analysis, revealing enhanced phosphorylation of the ROCK target MYPT1 at T696 in Ah-CRE; LSL-ROCK2:ER mouse intestinal epithelium and not in the Ah-CRE; LSL-KD2:ER tissues (Figure 4B). Taken together, these results demonstrate that $L S L-R O C K 2: E R$ mice inducibly express conditionally-active ROCK2 in a tissue-specific manner.

As a test of the consequences of conditional ROCK2 activation in the whole mouse, LSL-ROCK2:ER and LSL-KD2:ER mice were separately crossed with $\operatorname{Tg}\left(C A G-c r e / E s r 1^{*}\right) 5 A m c$ (CAG-CRE:ER) (Hayashi and McMahon, 2002) mice that 
undergo CRE-mediated recombination in most tissues in response to tamoxifen treatment (Figure 5A). When mice were administered tamoxifen citrate in their drinking water $(100 \mu \mathrm{g} / \mathrm{mL})$ to induce CRE-mediated recombination and ROCK2:ER fusion protein activation, 6/6 CAG-CRE:ER; LSL-ROCK2:ER mice died within 7 days, while all tamoxifen treated CAG-CRE:ER; LSL-KD2:ER and untreated CAGCRE:ER; LSL-ROCK2:ER mice survived over this time period (Figure 5B). When brains were dissected and examined histologically, it became apparent that all tamoxifen treated CAG-CRE:ER; LSL-ROCK2:ER mice had evidence of cerebral hemorrhagic lesions that were not observed in tamoxifen treated CAG-CRE:ER; $L S L-K D 2: E R$ (Figure $5 C$ ). These results suggest that there would be potential for the CAG-CRE:ER; LSL-ROCK2:ER transgenic mouse line to be used as an inducible cerebral stroke model.

Although loss-of-function genetically-modified mice have been useful in determining how specific targets may make essential contributions to various biological processes, there is also considerable value in gain-of-function (GoF) systems. Rather than inferring how a given protein might be involved from a loss of effect, GoF mutants can be used to determine biological roles by observing direct positive effects. We have described a GoF system that allows for the conditional activation of ROCK2 activity in a tissue selective manner, which has a wide-variety of potential applications for the study of tissue homeostasis and function, as well as in models of pathological conditions and diseases including cancer. 


\section{Methods}

\section{Animal models}

Conditional LSL-ROCK2:ER and control LSL-KD2:ER mice were constructed by targeting loxP-Stop-loxP ROCK2:ER or KD2:ER transgenes under the transcriptional control of a CAG promoter by gene-targeting each to the Hprt locus using an approach we have previously described (Samuel et al., 2009; Schachtner et al., 2012). The CAG loxP-Stop-loxP transgene was made by first replacing the SA region of the plasmid pBigT (gift of Frank Costantini; Addgene plasmid 21270) (Srinivas et al., 2001) with the chimeric synthetic promoter CAG (Niwa et al., 1991) from the plasmid pTurbo-CRE (a gift from Prof. Tim Ley, The Genome Center at Washington University, St. Louis, USA). The 3-phosphoglycerate kinase (PGK) promoter and EM7 promoter sequence from pL452 were then inserted into pBigT.CAG by recombineering in E. coli (Liu and Bambara, 2003). Short $5^{\prime}$ and $3^{\prime}$ homology arms from the Hprt targeting plasmid pSKB1 (Bronson et al., 1996) were placed flanking the expression cassette to generate pHprt.CAG.IoxP-STOP-loxP. ROCK2:ER or KD2:ER fusion protein cDNAs were then cloned downstream of the IoxP-Stop-IoxP $(L S L)$ transcriptional termination cassette. The transgene was then recombineered into pSKB1 (Bronson et al., 1996) to generate the final targeting pHprt-CAG-LSL-ROCK2:ER or pHprt-CAG-LSL-KD2:ER vectors.

The targeting vector was linearized and transferred into Hprt-deficient HM1 embryonic stem (ES) cells (Magin et al., 1992) by electroporation, which were then cultured on DR4 mouse embryonic fibroblast feeder layers (Tucker et al., 1997). Recombinants were selected in HAT supplement containing medium (Sigma) and appropriate targeting of the Hprt locus on $5^{\prime}$ and $3^{\prime}$ sides was confirmed by PCR using genomic DNA template prepared from HAT-resistant colonies. Genotyping 
used the Expand ${ }^{\mathrm{TM}}$ Long Template PCR system (Roche) according to the manufacturer's instructions. Primer pairs for genotyping the targeted ES cells were $5^{\prime}$ GTTGCTGAGGCAAAAATAGTGTAAT and CCATTTACCGTAAGTTATGTAACGC and $3^{\prime}$

CTACCTAGTGAGCCTGCAAACTG

and

\section{ATGTAAGTGCTAGGAATTGAACCTG.}

Following identification of correctly targeted clones, mouse lines were derived by injection into C57BL/6J blastocysts according to standard protocols (Nagy et al., 2003). Germline transgene transmission was identified by inspection of progeny coat colour and confirmed by PCR.

All procedures were performed under appropriate licenses and according to the UK Home Office guidelines or under approval granted by the SA Pathology/CALHN Animal Ethics Committee and according to Australian Code for the Care and Use of Animals for Scientific Purposes, 8th edition (2013). Mouse lines described herein will be made available to the research community.

\section{Polymerase chain reaction (PCR)}

$2.5 \mu \mathrm{l}$ of genomic DNA was used as a template in a $25 \mu \mathrm{l}$ reaction volume containing $2.5 \mathrm{mM} \mathrm{MgCl}_{2}, 0.5 \mu \mathrm{M}$ reverse and forward primers for each transgene, $0.25 \mathrm{mM}$ dNTPs and 1 unit Taq DNA polymerase $(1 \mathrm{U} / \mu \mathrm{l})$ in a $200 \mu \mathrm{l} \mathrm{PCR}$ tube. PCR was performed for each transgene in a thermal cycler. Electrophoresis was performed on the PCR products using $1 \%$ agarose gels that were stained with ethidium bromide. ROCK2 primers: Forward CGACCACTACCAGCAGAACA; Reverse GACGAACCAACTGCACTTCA. K14-CRE:PR primers: Forward CGGTCGATGCAACGAGTGAT; Reverse CCACCGTCAGTACGTGAGAT. 


\section{Keratinocyte isolation and culture}

Primary keratinocytes were extracted from tail skin as described previously (Samuel et al., 2011). Cells were maintained at $37^{\circ} \mathrm{C}$, with $5 \% \mathrm{CO}_{2}$ and $3 \% \mathrm{O}_{2}$, in keratinocyte growth medium (KGM-2; Lonza) and $50 \mu \mathrm{M} \mathrm{Ca}^{2+}$ on collagen-1 coated dishes (10 $\mu \mathrm{g} / \mathrm{mL}$; Advanced BioMatrix 5005).

\section{Small molecules}

4-Hydroxytamoxifen (Sigma H7904), RU486 (Sigma M8046), $\beta-N a p h t h o f l a v o n e$ (Sigma N3633), tamoxifen (Sigma T5648), tamoxifen citrate salt (Sigma T9262).

\section{Immunoblotting}

Standard protocols were used for Western blot analysis. Whole cell lysates were prepared in cell lysis buffer (1\% SDS (w/v), $50 \mathrm{mM}$ Tris $\mathrm{pH} 7.5)$ and protein concentration was determined by bicinchoninic assay (Sigma). ROCK1/2 (Millipore 07-1458) or GFP (Abcam ab6556) primary antibodies were used at 1:500 dilution. Alexa-Fluor 680-conjugated (Thermo Fisher Scientific) secondary antibody was detected by infrared imaging (Li-Cor Odyssey).

\section{Immunohistochemistry and immunofluorescence analysis}

Formalin-fixed, paraffin-embedded sections were rehydrated and immersed in 10 $\mathrm{mM}$ citric acid buffer at $\mathrm{pH} 6.0$, boiled for $20 \mathrm{~min}$, cooled, and sequentially blocked with $3 \% \mathrm{H}_{2} \mathrm{O}_{2}$ and $10 \%$ normal goat serum in PBS. Sections were then incubated with primary antibody, followed by incubation with Envision + System-HRP labeled Polymer (Dako). Primary antibodies were routinely used at $1: 50$ or $1: 100$ dilutions. Staining was visualized with Liquid DAB + Substrate (Dako). Immunofluorescence 
analysis was carried out as for immunohistochemistry, eliminating the peroxide blocking step. HRP-labeled secondary antibodies were replaced by Alexa Fluor 488 (green) or 594 (red)-conjugated secondary antibodies (Thermo Fisher Scientific) and slides were mounted in VECTASHIELD mounting medium (Vector Labs). Primary antibodies used: ROCK2 (Santa Cruz SC-5561), GFP (Clontech 632377), pMYPT1 (Thr696; Millipore ABS45), cytokeratin 14 (Leica Biosystems). Photomicrographs were obtained with a Zeiss microscope and Axiovision image capture software. Immunofluorescence images were acquired using an LSM 700 confocal system (Zeiss) or using a Leica SCN $400 f$ scanner and Leica Slidepath Digital Hub software, respectively.

\section{Acknowledgements}

Funding was from Cancer Research UK (A18276), NHMRC Australia (GNT1103712), Cancer Council SA and the Health Services Charitable Gifts Board, South Australia. Thanks to the Cancer Research UK Beatson Institute Histology and Biological Services, and staff of the SA Pathology Animal Care Facility.

\section{References}

Berton TR, Wang X-J, Zhou Z, Kellendonk C, Schütz G, Tsai S, Roop DR. 2000. Characterization of an inducible, epidermal-specific knockout system: Differential expression of lacZ in different Cre reporter mouse strains. genesis 26: $160-161$.

Bronson SK, Plaehn EG, Kluckman KD, Hagaman JR, Maeda N, Smithies O. 1996. Single-copy transgenic mice with chosen-site integration. Proc Natl Acad Sci USA 93: 9067-9072. 
Coleman M, Sahai E, Yeo M, Bosch M, Dewar A, Olson M. 2001. Membrane blebbing during apoptosis results from caspase-mediated activation of ROCK I. Nat Cell Biol 3: 339-345.

Croft D, Coleman M, Li S, Robertson D, Sullivan T, Stewart C, Olson M. 2005. Actinmyosin-based contraction is responsible for apoptotic nuclear disintegration. $J$ Cell Biol 168: 245-255.

Croft D, Sahai E, Mavria G, Li S, Tsai J, Lee W, Marshall C, Olson M. 2004. Conditional ROCK activation in vivo induces tumor cell dissemination and angiogenesis. Cancer Res 64: 8994-9001.

Croft DR, Olson MF. 2006a. Conditional Regulation of a ROCK-Estrogen Receptor Fusion Protein. Methods Enzymol 406: 541-553.

Croft DR, Olson MF. 2006b. The Rho GTPase Effector ROCK Regulates Cyclin A, Cyclin D1, and p27Kip1 Levels by Distinct Mechanisms. Mol Cell Biol 26: $4612-4627$.

Danielian PS, White R, Hoare SA, Fawell SE, Parker MG. 1993. Identification of residues in the estrogen receptor that confer differential sensitivity to estrogen and hydroxytamoxifen. Mol Endocrinol 7: 232-240.

Dassule HR, Lewis P, Bei M, Maas R, McMahon AP. 2000. Sonic hedgehog regulates growth and morphogenesis of the tooth. Development 127: 47754785.

Geeves MA. 2016. Review: The ATPase mechanism of myosin and actomyosin. Biopolymers 105: 483-491.

Hayashi S, McMahon AP. 2002. Efficient Recombination in Diverse Tissues by a Tamoxifen-Inducible Form of Cre: A Tool for Temporally Regulated Gene Activation/Inactivation in the Mouse. Dev Bio/244: 305-318. 
Ireland H, Kemp R, Houghton C, Howard L, Clarke AR, Sansom OJ, Winton DJ. 2004. Inducible cre-mediated control of gene expression in the murine gastrointestinal tract: effect of loss of $\beta$-catenin. Gastroenterology 126: 12361246.

Jackson EL, Willis N, Mercer K, Bronson RT, Crowley D, Montoya R, Jacks T, Tuveson DA. 2001. Analysis of lung tumor initiation and progression using conditional expression of oncogenic K-ras. Genes Dev 15: 3243-3248.

Julian L, Olson MF. 2014. Rho-associated coiled-coil containing kinases (ROCK). Small GTPases 5: e29846.

Kular J, Scheer KG, Pyne NT, Allam AH, Pollard AN, Magenau A, Wright RL, Kolesnikoff N, Moretti PA, Wullkopf L, Stomski FC, Cowin AJ, Woodcock JM, Grimbaldeston MA, Pitson SM, Timpson P, Ramshaw HS, Lopez AF, Samuel MS. 2015. A Negative Regulatory Mechanism Involving 14-3-3zeta Limits Signaling Downstream of ROCK to Regulate Tissue Stiffness in Epidermal Homeostasis. Dev Cell 35: 759-774.

Kumar MS, Hancock DC, Molina-Arcas M, Steckel M, East P, Diefenbacher M, Armenteros-Monterroso E, Lassailly F, Matthews N, Nye E, Stamp G, Behrens A, Downward J. 2012. The GATA2 transcriptional network is requisite for RAS oncogene-driven non-small cell lung cancer. Cell 149: 642655.

Kümper S, Mardakheh FK, McCarthy A, Yeo M, Stamp GW, Paul A, Worboys J, Sadok A, Jørgensen C, Guichard S, Marshall CJ. 2016. Rho-associated kinase $(\mathrm{ROCK})$ function is essential for cell cycle progression, senescence and tumorigenesis. eLife 5: e12203. 
Lee $\mathrm{DH}$, Shi J, Jeoung NH, Kim MS, Zabolotny JM, Lee SW, White MF, Wei L, Kim YB. 2009. Targeted disruption of ROCK1 causes insulin resistance in vivo. $J$ Biol Chem 284: 11776-11780.

Lochhead PA, Wickman G, Mezna M, Olson MF. 2010. Activating ROCK1 somatic mutations in human cancer. Oncogene 29: 2591-2598.

Magin TM, McWhir J, Melton DW. 1992. A new mouse embryonic stem cell line with good germ line contribution and gene targeting frequency. Nucleic Acids Res 20: $3795-3796$.

Niwa H, Yamamura K, Miyazaki J. 1991. Efficient selection for high-expression transfectants with a novel eukaryotic vector. Gene 108: 193-199.

Olson MF. 2008. Applications for ROCK kinase inhibiton. Curr Opin Cell Biol 20: $242-248$.

Olson MF. 2016. Rho GTPases, their post-translational modifications, diseaseassociated mutations and pharmacological inhibitors. Small GTPases: 1-13. doi: $10.1080 / 21541248.2016 .1218407$

Rath N, Olson MF. 2012. Rho-associated kinases in tumorigenesis: re-considering ROCK inhibition for cancer therapy. EMBO Rep 13: 900-908.

Sadok A, Marshall CJ. 2014. Rho GTPases: masters of cell migration. Small GTPases 5: e29710.

Sadok A, McCarthy A, Caldwell J, Collins I, Garrett MD, Yeo M, Hooper S, Sahai E, Kuemper S, Mardakheh FK, Marshall CJ. 2015. Rho kinase inhibitors block melanoma cell migration and inhibit metastasis. Cancer Res 75: 2272-2284.

Samuel MS, Lopez JI, McGhee EJ, Croft DR, Strachan D, Timpson P, Munro J, Schroeder E, Zhou J, Brunton VG, Barker N, Clevers H, Sansom OJ, Anderson KI, Weaver VM, Olson MF. 2011. Actomyosin-Mediated Cellular 
Tension Drives Increased Tissue Stiffness and beta-Catenin Activation to Induce Epidermal Hyperplasia and Tumor Growth. Cancer Cell 19: 776-791.

Samuel MS, Munro J, Bryson S, Forrow S, Stevenson D, Olson MF. 2009. Tissue selective expression of conditionally-regulated ROCK by gene targeting to a defined locus. genesis 47: 440-446.

Sanz-Moreno V, Gaggioli C, Yeo M, Albrengues J, Wallberg F, Viros A, Hooper S, Mitter R, Feral CC, Cook M, Larkin J, Marais R, Meneguzzi G, Sahai E, Marshall CJ. 2011. ROCK and JAK1 Signaling Cooperate to Control Actomyosin Contractility in Tumor Cells and Stroma. Cancer Cell 20: 229-245.

Satoh S, Ikegaki I, Kawasaki K, Asano T, Shibuya M. 2014. Pleiotropic effects of the rho-kinase inhibitor fasudil after subarachnoid hemorrhage: a review of preclinical and clinical studies. Curr Vasc Pharmacol 12: 758-765.

Schachtner H, Li A, Stevenson D, Calaminus SDJ, Thomas SG, Watson SP, Sixt M, Wedlich-Soldner R, Strathdee D, Machesky LM. 2012. Tissue inducible Lifeact expression allows visualization of actin dynamics in vivo and ex vivo. Eur J Cell Biol 91: 923-929.

Sebbagh M, Hamelin J, Bertoglio J, Solary E, Breard J. 2005. Direct cleavage of ROCK II by granzyme B induces target cell membrane blebbing in a caspaseindependent manner. J Exp Med 201: 465-471.

Selfridge J, Pow AM, McWhir J, Magin TM, Melton DW. 1992. Gene targeting using a mouse HPRT minigene/HPRT-deficient embryonic stem cell system: inactivation of the mouse ERCC-1 gene. Somat Cell Mol Genet 18: 325-336.

Shimizu Y, Thumkeo D, Keel J, Ishizaki T, Oshima H, Oshima M, Noda Y, Matsumura F, Taketo MM, Narumiya S. 2005. ROCK-I regulates closure of 
the eyelids and ventral body wall by inducing assembly of actomyosin bundles. J Cell Biol 168: 941-953.

Srinivas S, Watanabe T, Lin CS, William CM, Tanabe Y, Jessell TM, Costantini F. 2001. Cre reporter strains produced by targeted insertion of EYFP and ECFP into the ROSA26 locus. BMC Dev Biol 1: 4.

Thumkeo D, Shimizu Y, Sakamoto S, Yamada S, Narumiya S. 2005. ROCK-I and ROCK-II cooperatively regulate closure of eyelid and ventral body wall in mouse embryo. Genes Cells 10: 825-834.

Tucker KL, Wang Y, Dausman J, Jaenisch R. 1997. A transgenic mouse strain expressing four drug-selectable marker genes. Nucleic Acids Res 25: 37453746.

Uehata M, Ishizaki $\mathrm{T}$, Satoh $\mathrm{H}$, Ono $\mathrm{T}$, Kawahara $\mathrm{T}$, Morishita $\mathrm{T}$, Tamakawa $\mathrm{H}$, Yamagami K, Inui J, Maekawa M, Narumiya S. 1997. Calcium sensitization of smooth muscle mediated by a Rho-associated protein kinase in hypertension. Nature 389: 990-994.

Wagner K-U, Wall RJ, St-Onge L, Gruss P, Wynshaw-Boris A, Garrett L, Li M, Furth PA, Hennighausen L. 1997. Cre-mediated gene deletion in the mammary gland. Nucleic Acids Res 25: 4323-4330.

Yap TA, Walton MI, Grimshaw KM, te Poele RH, Eve PD, Valenti MR, de Haven Brandon AK, Martins V, Zetterlund A, Heaton SP, Heinzmann K, Jones PS, Feltell RE, Reule M, Woodhead SJ, Davies TG, Lyons JF, Raynaud FI, Eccles SA, Workman P, Thompson NT, Garrett MD. 2012. AT13148 Is a Novel, Oral Multi-AGC Kinase Inhibitor with Potent Pharmacodynamic and Antitumor Activity. Clin Cancer Res 18: 3912-3923. 
Zhang Y-MM, Bo J, Taffet GE, Chang J, Shi J, Reddy AK, Michael LH, Schneider MD, Entman ML, Schwartz RJ, Wei L. 2006. Targeted deletion of ROCK1 protects the heart against pressure overload by inhibiting reactive fibrosis. FASEB J 20: 916-925.

\section{Figure Legends}

Figure 1. Targeting a conditionally-active loxP-Stop-loxP ROCK2:ER transgene to the Hprt locus. (A) The conditionally-active ROCK2:ER fusion protein is inactive in the absence of ligand. Upon stimulation with estrogen analogues including 4hydroxytamoxifen $(4 \mathrm{HT})$, the specific activity of the kinase domain of ROCK2 increases leading to phosphorylation of substrates including MYPT1, MLC and LIM kinases 1 and 2 (LIMK1/2), which results in the generation of actomyosin contractile force. $E G F P=$ enhanced green fluorescent protein. $E R=$ modified hormone binding domain of the estrogen receptor. (B) The Hprt locus in mouse HM1 embryonic stem cells lacks a promoter and first two exons, allowing for targeting by the homology arms of the pHprt-CAG-LSL-ROCK2:ER plasmid that includes a promoter and first exon of human HPRT (yellow) and second exon of mouse Hprt. Successful targeting and homologous recombination reconstitutes an active Hprt locus that allows for positive selection with HAT medium. (C) By crossing with mice expressing CRE recombinase from tissue-selective promoters, CRE-mediated recombination between loxP sites removes the transcription termination cassette (STOP) to allow ROCK2:ER transgene expression.

Figure 2. Validation of $L S L-R O C K 2: E R$ expression in mouse skin. (A) $L S L-$ ROCK2:ER mice were crossed to a line expressing a CRE fusion with the hormone- 
binding domain of the progesterone receptor (PR) from a $K 14$ promoter. Treatment with the progesterone ligand RU486 enables recombination and consequent ROCK2:ER expression. Stimulation with 4-hydroxytamoxifen then stimulates ROCK2:ER catalytic activity. (B) PCR analysis of the ROCK2:ER and CRE transgenes in mouse ear skin without or with RU486 treatment. Unrecombined LSLROCK2:ER results in the production of a 575 bp PCR band in all samples, while a 600 bp CRE:PR is seen only in the 4 transgene expressing mice. Following RU486 treatment, recombination resulted in the production of a $500 \mathrm{bp}$ PCR band, indicating dependence on both CRE expression and RU486 treatment. (C, D) Validation of LSL-ROCK2:ER recombination and consequent ROCK2:ER expression in mouse skin following topical RU486 treatment by EGFP or ROCK2 immunohistochemical staining. Scale bar $=100 \mu \mathrm{m}$. (E) Treatment with RU486 does not lead to increased MYPT1 phosphorylation (pMYPT1) unless combined with topical 4HT treatment to conditionally activate ROCK2:ER. (F) Validation of LSL-ROCK2:ER expression in epidermal keratinocytes following crossing with K14-CRE expressing mice. Primary keratinoctyes were isolated from the tails of mice with $L S L-R O C K 2: E R$ alone, $L S L-$ ROCK2:ER plus K14-CRE or K14-ROCK2:ER as indicated. A ROCK2:ER protein band was only detected by blotting with ROCK2 or GFP antibodies in keratinocytes from LSL-ROCK2:ER plus K14-CRE or positive control K14-ROCK2:ER mice. Consistent with previous observations, treatment with $4 \mathrm{HT}$ increased ROCK2:ER protein levels relative to untreated cells.

Figure 3. Validation of $L S L-R O C K 2: E R$ expression in mouse mammary epithelium. (A) LSL-ROCK2:ER and LSL-KD2:ER mice were crossed separately onto a strain expressing a CRE recombinase under the control of the MMTV promoter. Mice will 
express the ROCK2:ER or KD2:ER fusion protein constitutively, but transgenederived ROCK will not be activated until systemic treatment with tamoxifen, which is metabolized to $4 \mathrm{HT}$ and activates ROCK2:ER but not kinase-dead KD2:ER. (B) Immunofluorescence analysis of mammary tissues derived from $L S L-K D 2: E R, L S L-$ ROCK2:ER, MMTV-CRE; LSL-KD2:ER or MMTV-CRE; LSL-ROCK2:ER mice showing expression of the EGFP:KD2:ER or EGFP:ROCK2:ER fusion proteins in CRE-expressing mice. Scale bars $=50 \mu \mathrm{m}$ (C) Immunofluorescence analysis (cytokeratin 14 [white in monochromatic panels and red in Merge] to mark basal mammary epithelium and PMYPT1 [white in monochromatic panels and green in Merge]) of mammary tissue derived from LSL-KD2:ER, LSL-ROCK2:ER, MMTVCRE; LSL-KD2:ER and MMTV-CRE; LSL-ROCK2:ER mice that have been treated systemically with tamoxifen, showing elevated phosphorylation of MYPT1 in CREexpressing $L S L-R O C K 2: E R$ mammary epithelium but not $L S L-K D 2: E R$ expressing tissues. Scale bars $=50 \mu \mathrm{m}$.

Figure 4. Validation of $L S L-R O C K 2: E R$ expression in mouse intestinal epithelium. (A) LSL-ROCK2:ER and LSL-KD2:ER mice were crossed to a line expressing a CRE recombinase under the control of the cytochrome P450 Cyp1A1 (Ah) promoter. Mice will express the ROCK2:ER or KD2:ER fusion protein upon activation of the Cyp1A1 promoter by systemic treatment with $\beta$-Naphthoflavone, but transgene-derived ROCK will not be activated until systemic treatment with tamoxifen, which is metabolized to $4 \mathrm{HT}$ and activates ROCK2:ER. (B) Immunofluorescence analysis (pMYPT1 [green]) of intestinal epithelium derived from Ah-CRE; LSL-KD2:ER and Ah-CRE; LSL-ROCK2:ER mice that have been treated systemically with tamoxifen, 
showing elevated phosphorylation of MYPT1 in CRE-expressing LSL-ROCK2:ER intestinal epithelium but not $L S L-K D 2: E R$ tissues.

Figure 5. Consequence of conditional activation of ROCK2:ER in whole mice. (A) LSL-ROCK2:ER and LSL-KD2:ER mice were crossed to a line expressing a CRE:ER fusion recombinase under the control of an artificial chicken actin gene (CAG) promoter. Mice express the ROCK2:ER or KD2:ER fusion protein in a tamoxifeninduced manner in most tissues. Systemic treatment with tamoxifen, which is metabolized to $4 \mathrm{HT}$, activates ROCK2:ER but not KD2:ER fusion protein. (B) Survival curves for CAG-CRE:ER; LSL-ROCK2:ER and CAG-CRE:ER; LSL-KD2:ER mice that were untreated or treated with tamoxifen as indicated. Survival $p$-value determined by Mantel-Cox test (CAG-CRE:ER; LSL-ROCK2:ER untreated $\mathrm{n}=3$; CAG-CRE:ER; LSL-ROCK2:ER; tamoxifen treated $\mathrm{n}=6$ : CAG-CRE:ER; LSL$K D 2: E R$ untreated $\mathrm{n}=3$ ). (C) Brain sections from tamoxifen treated CAG-CRE:ER; LSL-KD2:ER (left) and CAG-CRE:ER; LSL-ROCK2:ER (middle) mice. Scale bars = 1 $\mathrm{mm}$. Red and blue rectangle insets indicate sites of cerebral hemorrhagic lesions in the CAG-CRE:ER; LSL-ROCK2:ER image are shown at higher magnification at right with corresponding borders. Scale bars $=100 \mu \mathrm{m}$. 
A
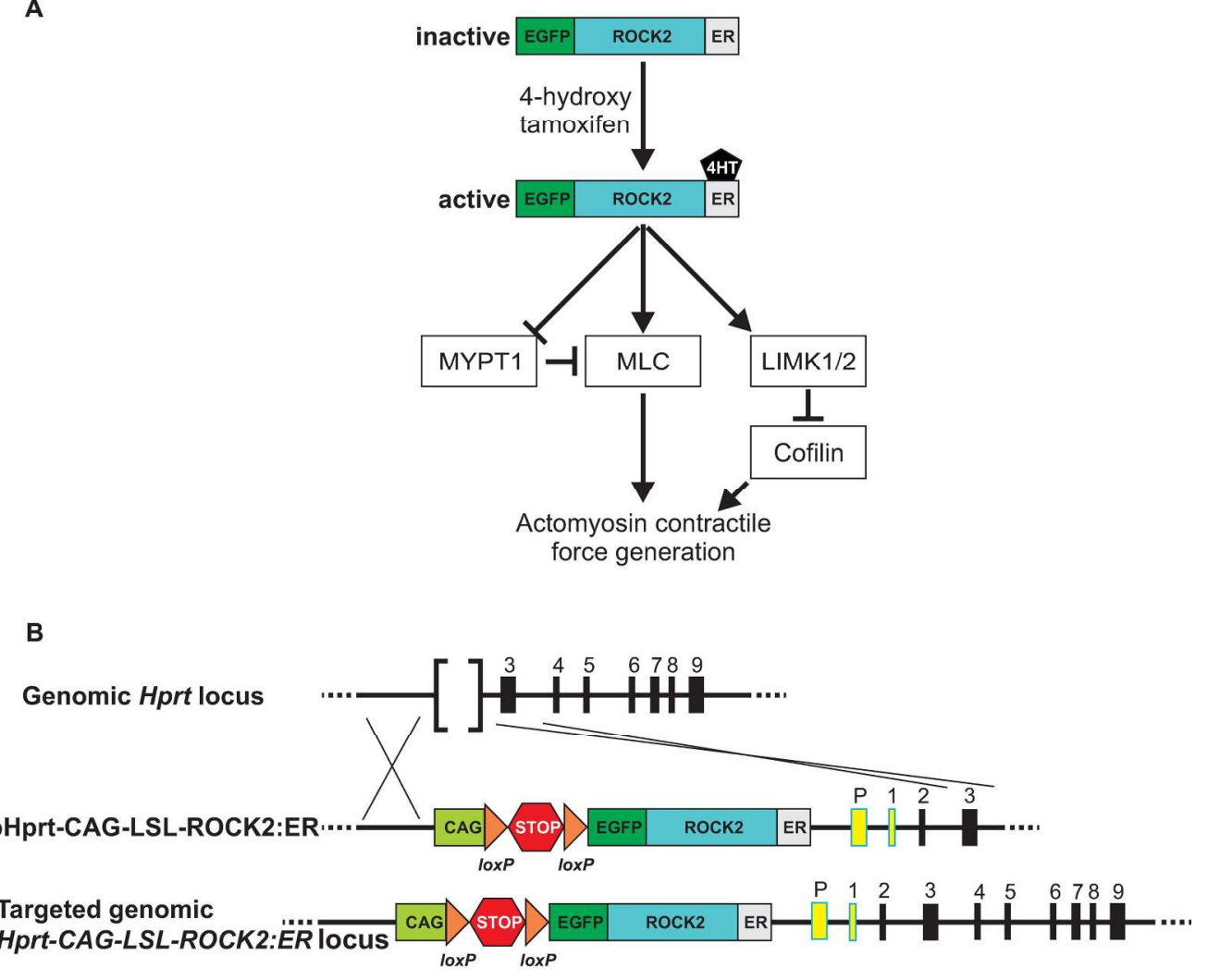

C

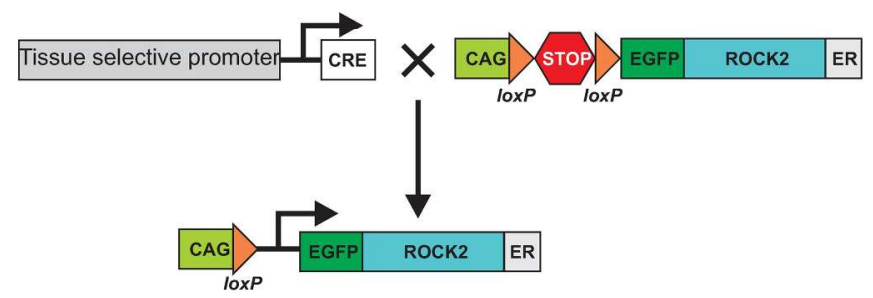

Figure 1. Targeting a conditionally-active loxP-Stop-loxP ROCK2:ER transgene to the Hprt locus. (A) The conditionally-active ROCK2:ER fusion protein is inactive in the absence of ligand. Upon stimulation with estrogen analogues including 4-hydroxytamoxifen (4HT), the specific activity of the kinase domain of ROCK2 increases leading to phosphorylation of substrates including MYPT1, MLC and LIM kinases 1 and 2 $($ LIMK1/2), which results in the generation of actomyosin contractile force. EGFP = enhanced green fluorescent protein. $E R=$ modified hormone binding domain of the estrogen receptor. (B) The Hprt locus in mouse HM1 embryonic stem cells lacks a promoter and first two exons, allowing for targeting by the homology arms of the pHprt-CAG-LSL-ROCK2:ER plasmid that includes a promoter and first exon of human HPRT (yellow) and second exon of mouse Hprt. Successful targeting and homologous recombination reconstitutes an active Hprt locus that allows for positive staining with HAT medium. (C) By crossing with mice expressing CRE recombinase from tissue-selective promoters, CRE-mediated recombination between loxP sites removes the transcription terminationcassette (STOP) to allow ROCK2:ER transgene expression.

Figure 1

$223 \times 250 \mathrm{~mm}(300 \times 300 \mathrm{DPI})$

John Wiley \& Sons, Inc.

This article is protected by copyright. All rights reserved. 
A

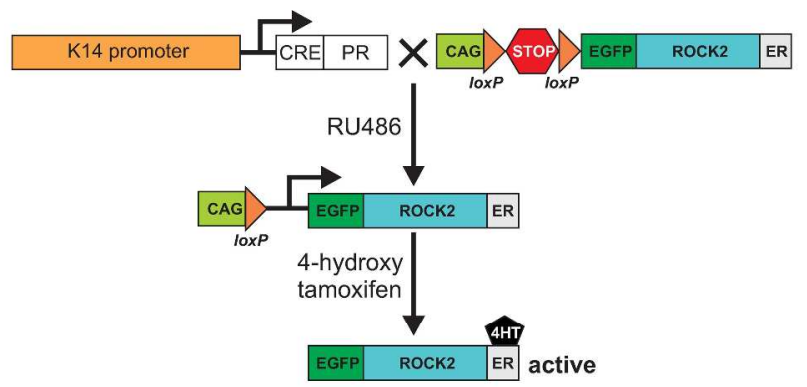

B

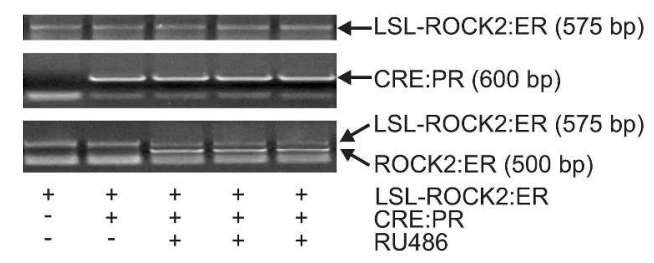

c

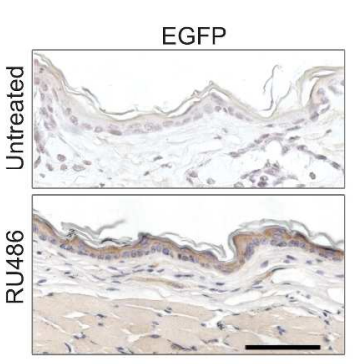

D

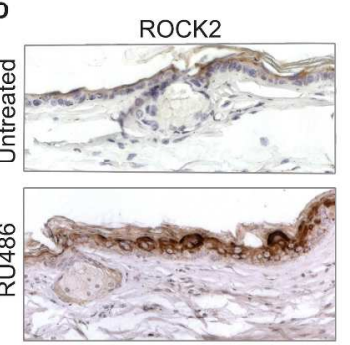

E
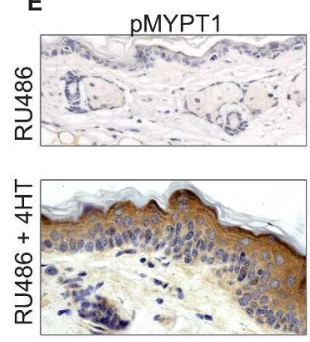

$\mathbf{F}$

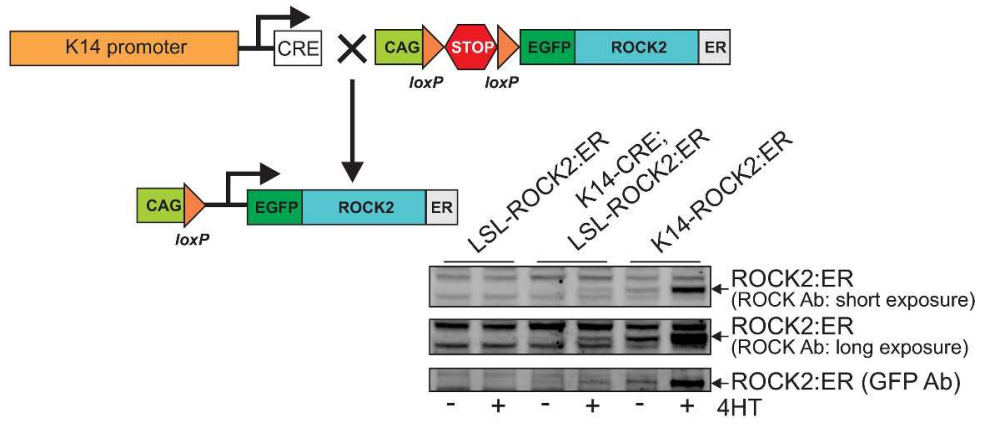

Figure 2. Validation of LSL-ROCK2:ER expression in mouse skin. (A) LSL-ROCK2:ER mice were crossed to a line expressing a CRE fusion with the hormone-binding domain of the progesterone receptor (PR) from a K14 promoter. Treatment with the progesterone ligand RU486 enables recombination and consequent ROCK2:ER expression. Stimulation with 4-hydroxytamoxifen then stimulates ROCK2:ER catalytic activity. (B) PCR analysis of the ROCK2:ER and CRE transgenes in mouse ear skin without or with RU486 treatment. Unrecombined LSL-ROCK2:ER results in the production of a 575 bp PCR band in all samples, while a 600 bp CRE:PR is seen only in the 4 transgene expressing mice. Following RU486 treatment, recombination resulted

in the production of a 500 bp PCR band, indicating dependence on both CRE expression and RU486 treatment. (C, D) Validation of LSL-ROCK2:ER recombination and consequent ROCK2:ER expression in mouse skin following topical RU486 treatment by EGFP or ROCK2 immunohistochemical staining. Scale bar $=100 \mu \mathrm{m}$. (E) Treatment with RU486 does not lead to increased MYPT1 phosphorylation (pMYPT1) unless combined with topical 4HT treatment to conditionally activate ROCK2:ER. (F) Validation of LSL-ROCK2:ER expression in epidermal keratinocytes following crossing with K14-CRE expressing mice. Primary 
keratinoctyes were isolated from the tails of mice with LSL-ROCK2:ER alone, LSL-ROCK2:ER plus K14-CRE or K14-ROCK2:ER as indicated. A ROCK2:ER protein band was only detected by blotting with ROCK2 or GFP antibodies in keratinocytes from LSL-ROCK2:ER plus K14-CRE or positive control K14-ROCK2:ER mice. Consistent with previous observations, treatment with $4 \mathrm{HT}$ increased ROCK2:ER protein levels relative to untreated cells.

Figure 2

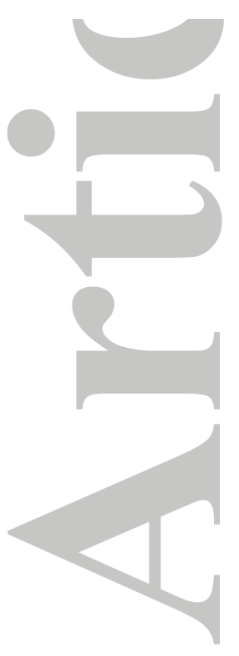

$274 \times 384 \mathrm{~mm}(300 \times 300$ DPI $)$

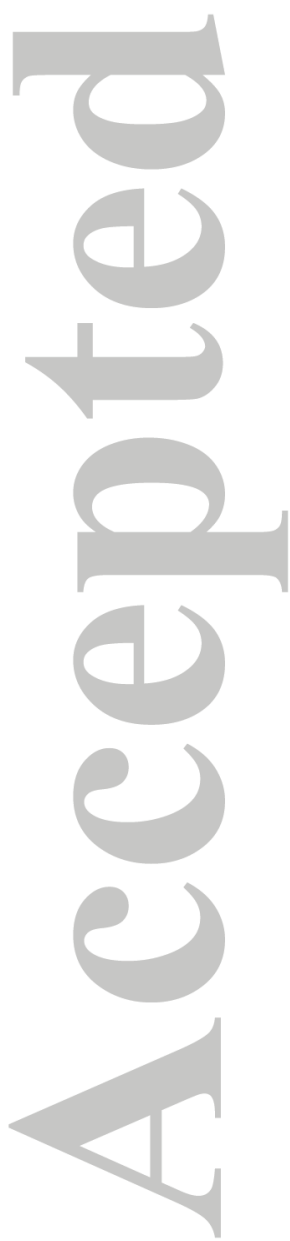

John Wiley \& Sons, Inc.

This article is protected by copyright. All rights reserved. 
A

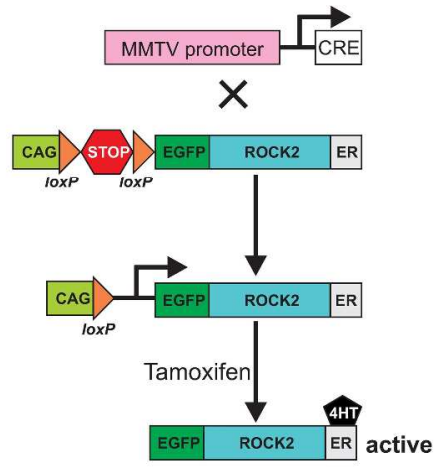

B

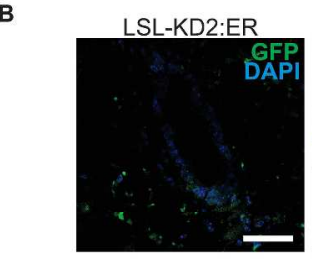

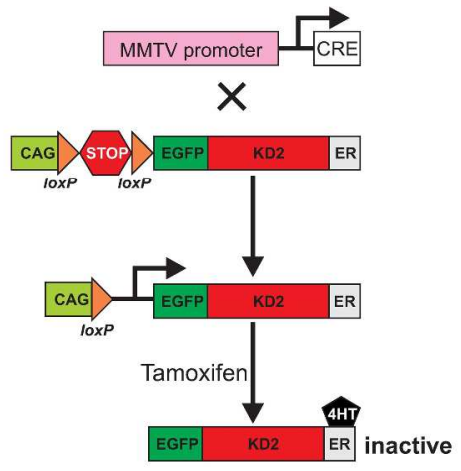

MMTV-Cre; LSL-KD2:ER

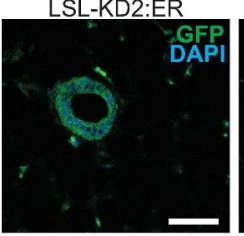

MMTV-Cre

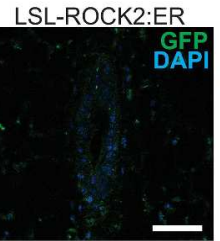

c
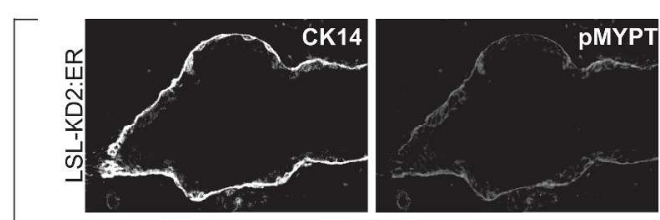

Merge
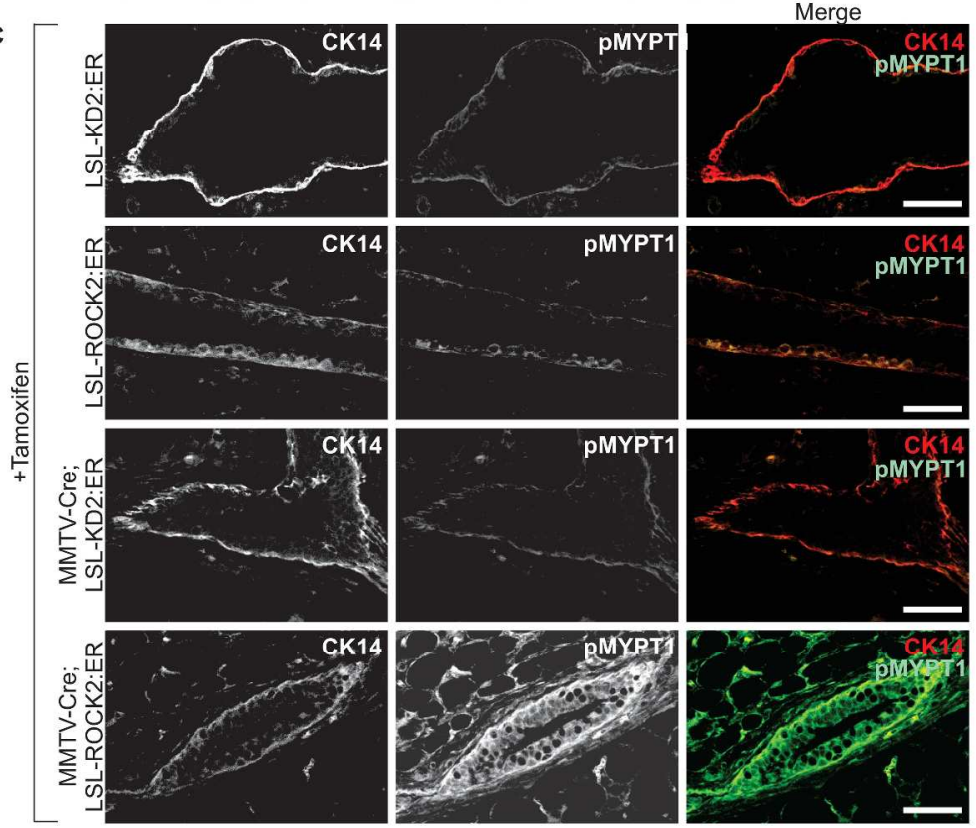

Figure 3. Validation of LSL-ROCK2:ER expression in mouse mammary epithelium. (A) LSL-ROCK2:ER and LSL-KD2:ER mice were crossed separately onto a strain expressing a CRE recombinase under the control of the MMTV promoter. Mice will express the ROCK2:ER or KD2:ER fusion protein constitutively, but transgenederived ROCK will not be activated until systemic treatment with tamoxifen, which is metabolized to $4 \mathrm{HT}$ and activates ROCK2:ER but not kinase-dead KD2:ER. (B) Immunofluorescence analysis of mammary tissues derived from LSL-KD2:ER, LSL-ROCK2:ER, MMTV-CRE; LSL-KD2:ER or MMTV-CRE; LSL-ROCK2:ER mice showing expression of the EGFP:KD2:ER or EGFP:ROCK2:ER fusion proteins in CRE-expressing mice. Scale bars $=50 \mu \mathrm{m}(\mathrm{C})$ Immunofluorescence analysis (cytokeratin 14 [white in monochromatic panels and red in

Merge] to mark basal mammary epithelium and pMYPT1 [white in monochromatic panels and green in Merge]) of mammary tissue derived from LSL-KD2:ER, LSL-ROCK2:ER, MMTV-CRE; LSL-KD2:ER and MMTV-

CRE; LSL-ROCK2:ER mice that have been treated systemically with tamoxifen, showing elevated phosphorylation of MYPT1 in CRE-expressing LSL-ROCK2:ER mammary epithelium but not LSL-KD2:ER expressing tissues. Scale bars $=50 \mu \mathrm{m}$.

Figure 3

$279 \times 427 \mathrm{~mm}(300 \times 300 \mathrm{DPI})$

John Wiley \& Sons, Inc.

This article is protected by copyright. All rights reserved. 
A
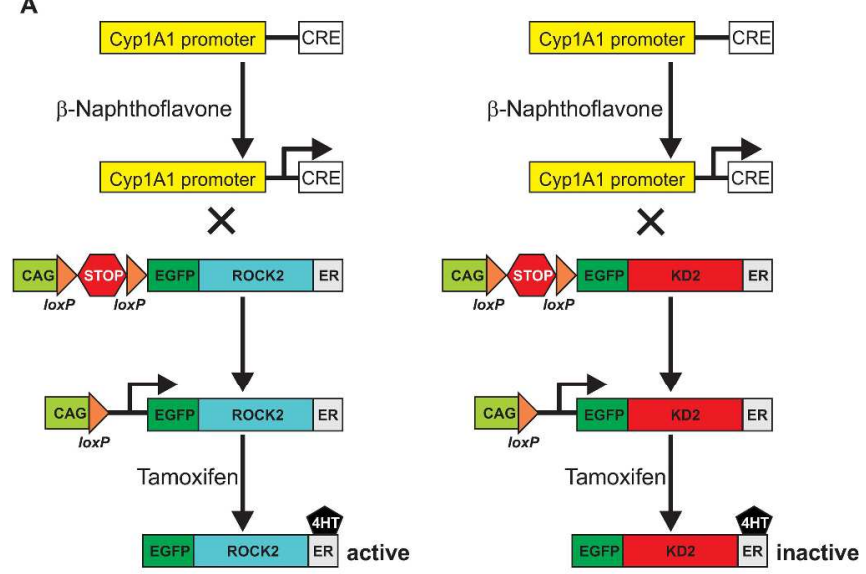

$\mathbf{B}$

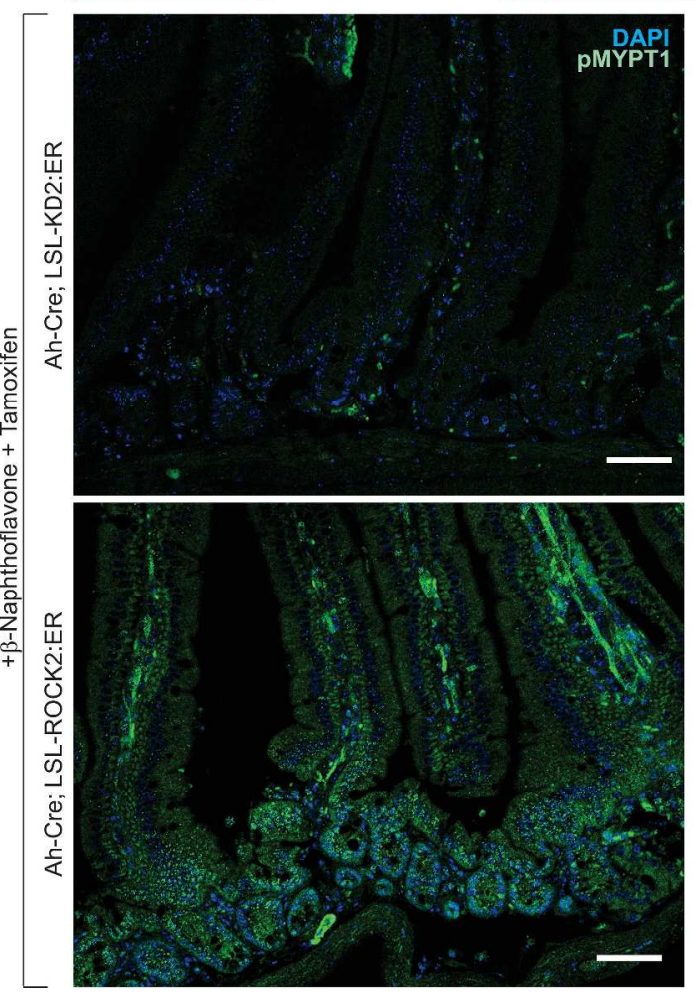

Figure 4. Validation of LSL-ROCK2:ER expression in mouse intestinal epithelium. (A) LSL-ROCK2:ER and LSL-KD2:ER mice were crossed to a line expressing a CRE recombinase under the control of the cytochrome P450 Cyp1A1 (Ah) promoter. Mice will express the ROCK2:ER or KD2:ER fusion protein upon activation of the Cyp1A1 promoter by systemic treatment with $\beta$-Naphthoflavone, but transgene-derived ROCK will not be activated until systemic treatment with tamoxifen, which is metabolized to $4 \mathrm{HT}$ and activates ROCK2:ER. (B) Immunofluorescence analysis (pMYPT1 [green]) of intestinal epithelium derived from Ah-CRE; LSL-KD2:ER and Ah-CRE; LSL-ROCK2:ER mice that have been treated systemically with tamoxifen, showing elevated phosphorylation of MYPT1 in CRE-expressing LSL-ROCK2:ER intestinal epithelium but not LSL-KD2:ER

tissues.

Figure 4

$273 \times 504 \mathrm{~mm}(300 \times 300 \mathrm{DPI})$

John Wiley \& Sons, Inc.

This article is protected by copyright. All rights reserved. 
A

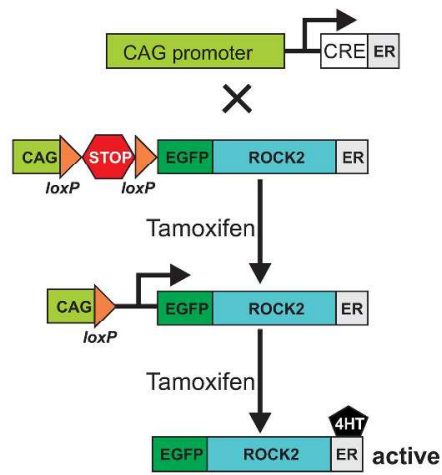

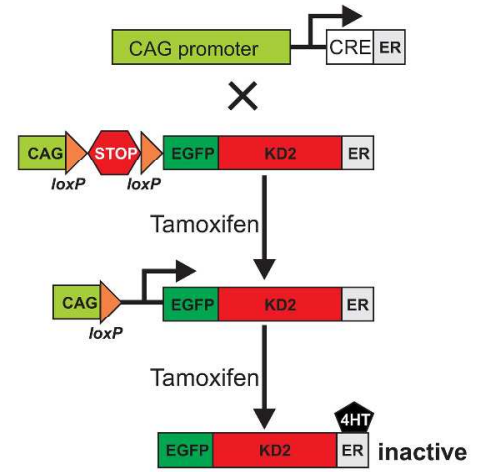

B

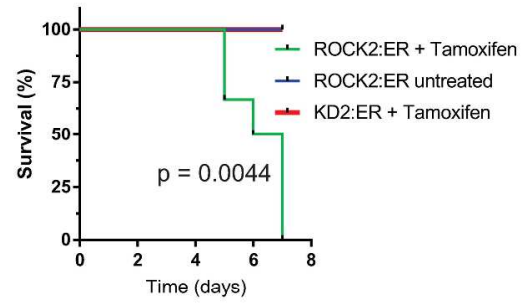

C CAG-Cre; LSL-KD2:ER + Tamoxifen

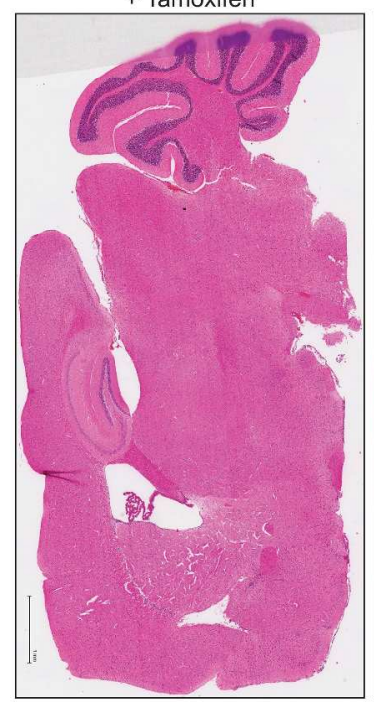

CAG-Cre; LSL-ROCK2:ER + Tamoxifen

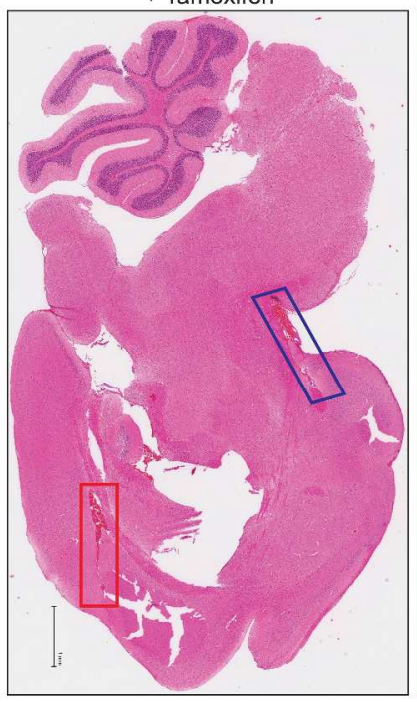

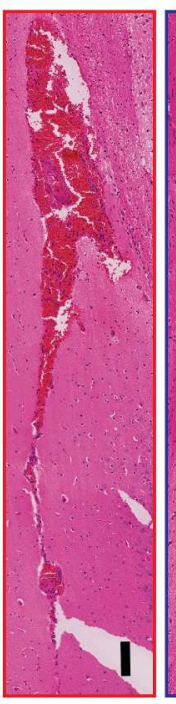

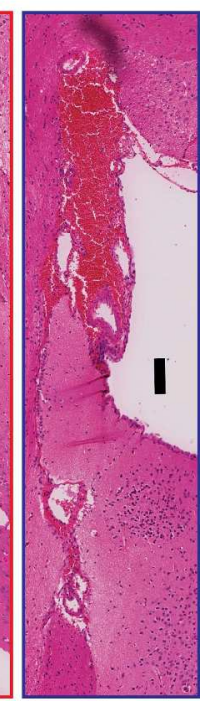

Figure 5. Consequence of conditional activation of ROCK2:ER in whole mice. (A) LSL-ROCK2:ER and LSLKD2:ER mice were crossed to a line expressing a CRE:ER fusion recombinase under the control of an artificial chicken actin gene (CAG) promoter. Mice express the ROCK2:ER or KD2:ER fusion protein in a tamoxifen-induced manner in most tissues. Systemic treatment with tamoxifen, which is metabolized to

$4 \mathrm{HT}$, activates ROCK2:ER but not KD2:ER fusion protein. (B) Survival curves for CAG-CRE:ER; LSL-

ROCK2:ER and CAG-CRE:ER; LSL-KD2:ER mice that were untreated or treated with tamoxifen as indicated. Survival $p$-value determined by Mantel-Cox test (CAG-CRE:ER; LSL-ROCK2:ER untreated $n=3$; CAGCRE:ER; LSL-ROCK2:ER; tamoxifen treated $n=6$ : CAG-CRE:ER; LSL-KD2:ER untreated $n=3)$. (C) Brain sections from tamoxifen treated CAG-CRE:ER; LSL-KD2:ER (left) and CAG-CRE:ER; LSL-ROCK2:ER (right) mice. Scale bars $=1 \mathrm{~mm}$. Red and blue rectangle insets indicate sites of cerebral haemorrhagic lesions in the CAG-CRE:ER; LSL-ROCK2:ER image are shown at higher magnification at right with corresponding borders. Scale bars $=100 \mu \mathrm{m}$.

Figure 5 